\title{
Use of Matlab package for external local calibration of IR camera with microbolometric FPA detector
}

\author{
by T. Kruczek*, W. Adamczyk* and G. Kruczek*
}

\author{
*Institute of Thermal Technology, Silesian University of Technology, 44-100 Gliwice, Poland, \\ tadeusz.kruczek@polsl.pl \\ *Institute of Thermal Technology, Silesian University of Technology, 44-100 Gliwice, Poland, \\ wojciech.adamczyk@polsl.pl \\ *Institute of Thermal Technology, Silesian University of Technology, 44-100 Gliwice, Poland, PhD student, \\ grzegorz.kruczek@polsı.pl
}

\begin{abstract}
This work presents the method of improving the accuracy of temperature measurement results obtained by means of long-wave infrared camera equipped with focal plane array detector. The typical accuracy of infrared cameras specified by their manufacturers amounts to $\pm 2 \mathrm{~K}$ or $\pm 2 \%$ of the measured value of temperature. Very often a better accuracy of the measurements is required, e.g. during the measurements of human body temperature. To improve the measurement accuracy an external correction of obtained results on the basis of comparison measurement of black body temperature and next with use of MATLAB package has been proposed.
\end{abstract}

\section{Nomenclature}

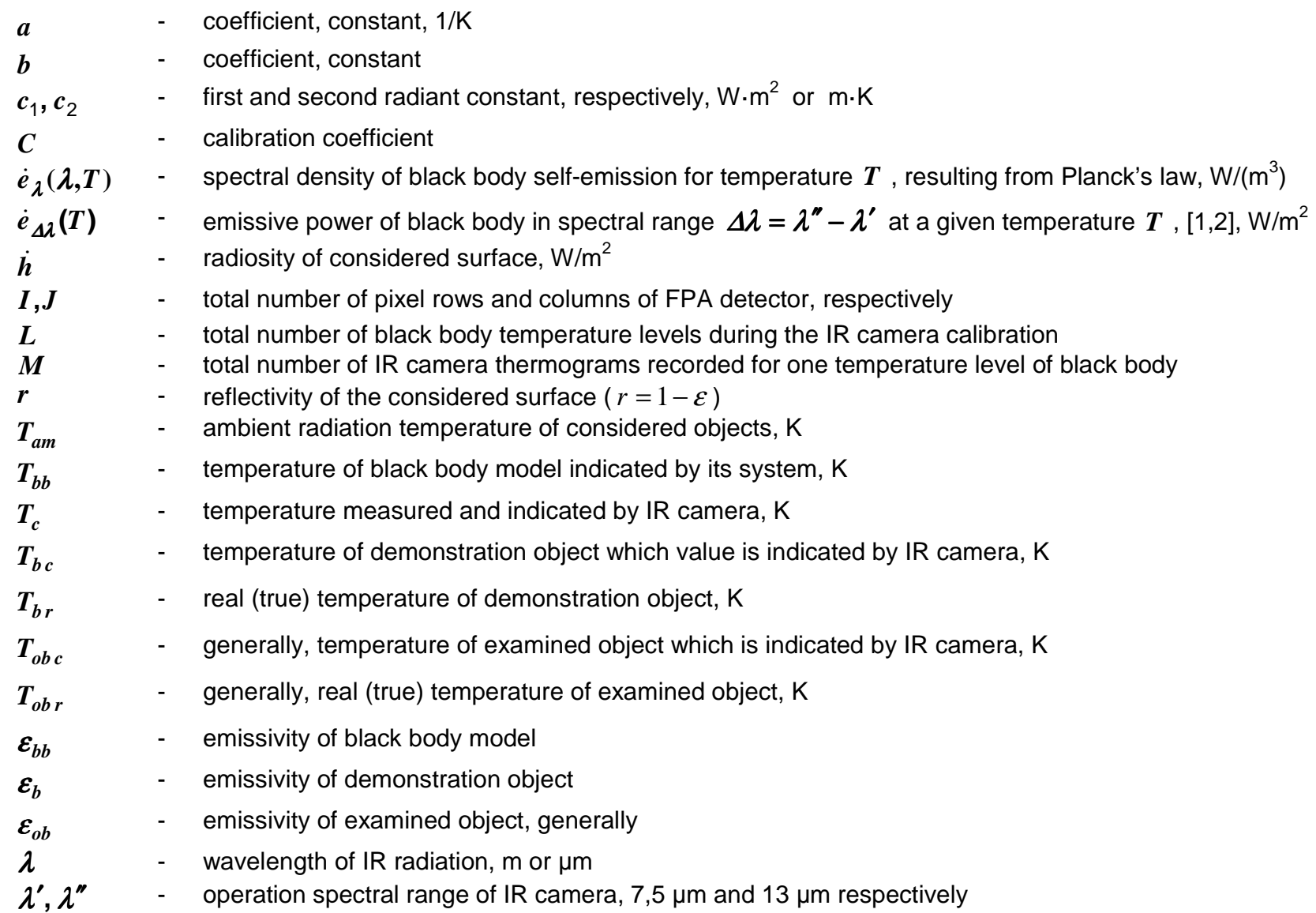

\section{Indexes and abbreviations}

$i, j \quad$ - consecutive number of row and column of FPA detector, respectively 


\subsection{1/qirt.2016.066}

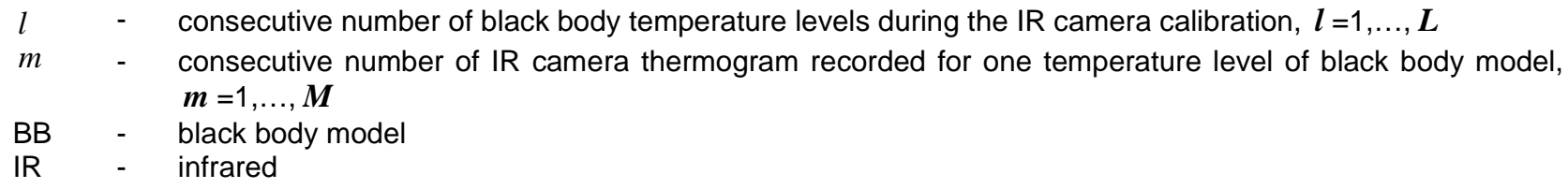

\section{Introduction}

The given typical values of measurement accuracy amounting to $\pm 2 \mathrm{~K}$ or $\pm 2 \%$ of the measured value are valid for the whole temperature measurement range of considered camera [3, 4]. The specified accuracy deals with the measurement results displayed directly by the camera. Simultaneously with the relentless passage of time the material of camera detector goes off and arises the problem of detector temperature drift which is of non-uniform character over the detector surface [5]. Therefore, to keep high accuracy of infrared camera measurements, the camera detector should be periodically calibrated [6].

In the case of measuring the temperature occurring within a narrow range of its values it is possible to improve the accuracy of obtained measurement results by means of external correction of raw measurement results obtained directly from the infrared camera. This correction can be done locally for aforementioned measurement range which should be determined at the beginning of this procedure.

In this methodology the using of a model of black body is necessary. The first step in this procedure is the measuring of the temperature of black body by means of the examined infrared camera. These measurements are repeated several times for different values of black body. These temperature values should cover the whole temperature range which is interested for us. Next, for each pixel of FPA (Focal Plane Array) detector the discrepancy between the real value of the black body temperature and value indicated by the infrared camera is determined. As the result, the matrixes of the discrepancies between the temperatures indicated by the camera and the temperature values reached by the black body model are obtained.

Then the gathered data were applied for the development of mathematical relationships used for the correction of measurement results produced by the examined camera.

\section{Description of calibration algorithm}

The infrared thermography technique is based on the measurement of radiative heat flux emitted from the surface taken into consideration. In the situation when the emissivity of the investigated object is less than $100 \%$, the total radiative heat flux from the tested surface consists of two parts. The first part presents the self-emission heat flux whereas the second part is the radiation flux which comes from surroundings and is reflected by the considered surface. Thus, in a mathematical model during an infrared camera temperature measurement the following radiation heat fluxes arriving at an infrared detector should be taken into account:

a) radiation flux emitted by the examined object,

b) radiation flux emitted by the ambient and reflected from the examined object,

c) radiation flux emitted by the atmospheric air which is to be found between the object and the infrared camera.

During the calibration procedure, due to a very small distance between the source of thermal radiation (a black body model) and the IR camera, the radiation flux specified in point (c) can be omitted. In this case a scheme of radiative heat transfer between considered object, its ambient elements and the IR camera can be shown by Fig. 1.

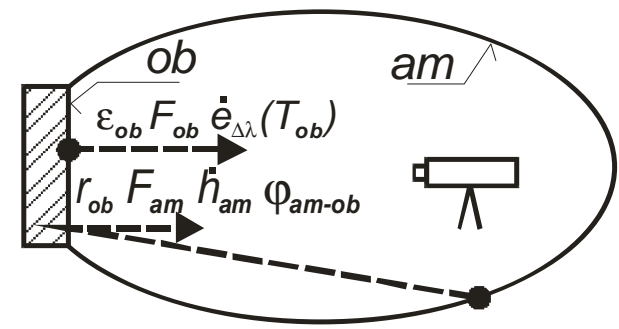

Fig. 1. Scheme of heat transfer in measurement space; ob-surface of object under investigation, am- isothermal ambient.

The quantity denoted by $\dot{e}_{\Delta \lambda}(T)$ presents a self-emission radiation heat flux emitted within the operation spectral range of the IR camera and is expressed by the relation:

$$
\dot{e}_{\Delta \lambda}(T)=\int_{\lambda^{\prime}}^{\lambda^{\prime \prime}} \dot{e}_{\lambda}(\lambda, T) \mathrm{d} \lambda
$$




\subsection{1/qirt.2016.066}

where $\dot{e}_{\lambda}(\lambda, T)$ is spectral density of black body self-emission resulting from Planck's law [1, 2], expressed by the equation (2):

$$
\dot{e}_{\lambda}(\lambda, T)=\frac{c_{1} \lambda^{-5}}{\exp \left(c_{2} / \lambda T\right)-1}
$$

The sum of self-emission heat flux and radiation heat fluxes coming from other surfaces and reflected by the surface under consideration is called the radiosity (denoted by $\dot{\boldsymbol{h}}$ ) of the considered surface [1, 2].

After applying the closure principle $\boldsymbol{\varphi}_{a m-o b}+\boldsymbol{\varphi}_{a m-a m}=1$ and reciprocity principle $\boldsymbol{F}_{o b} \boldsymbol{\varphi}_{o b-a m}=\boldsymbol{F}_{a m} \boldsymbol{\varphi}_{a m-o b}$, $[1,2]$, and after making obvious assumptions that $\boldsymbol{\varepsilon}_{a m}=1, \boldsymbol{\varphi}_{o b-a m}=1$, after some manipulations an accurate relation expressing the unit flux of radiation energy $\dot{\boldsymbol{h}}_{o b}$ coming from surface "ob" and arriving at an IR camera is obtained $[7,8,9]$ :

$$
\dot{h}_{o b}=\varepsilon_{o b} \dot{e}_{\Delta \lambda}\left(T_{o b}\right)+\left(1-\varepsilon_{o b}\right) \dot{e}_{\Delta \lambda}\left(T_{a m}\right)
$$

The output signal $s$ from the camera detector is closely coupled with the aforementioned energy flux and can be described by the formula [3, 7]:

$$
s \approx \psi \dot{h}_{o b}=\psi\left[\varepsilon_{o b} \dot{e}_{\Delta \lambda}\left(T_{o b}\right)+\left(1-\varepsilon_{o b}\right) \dot{e}_{\Delta \lambda}\left(T_{a m}\right)\right]
$$

During the calibration procedure the equation (4) is formulated twice for each $\boldsymbol{i}, \boldsymbol{j}$-th pixel of FPA detector. The first time for the IR camera with a detector correctly processing the radiation flux generated by black body model at temperature $\boldsymbol{T}_{\boldsymbol{b} \boldsymbol{b}}$ into output signal $\boldsymbol{s}_{\boldsymbol{c o r}}$ :

$$
s_{c o r} \approx \psi\left[\varepsilon_{b b} \dot{e}_{\Delta \lambda}\left(T_{b b}\right)+\left(1-\varepsilon_{b b}\right) \dot{e}_{\Delta \lambda}\left(T_{a m}\right)\right]
$$

and the second time for the IR camera with a detector which incorrectly converts the radiation flux generated by black body model at temperature $\boldsymbol{T}_{\boldsymbol{b} \boldsymbol{b}}$ into output signal $\boldsymbol{s}_{\text {incor }}$ and camera indicates an incorrect temperature value $\boldsymbol{T}_{\boldsymbol{c} i, j}$ for $\boldsymbol{i}, \boldsymbol{j}$-th detector pixel:

$$
s_{\text {incor }} \approx \psi\left[\varepsilon_{b b} \dot{e}_{\Delta \lambda}\left(T_{c i, j}\right)+\left(1-\varepsilon_{b b}\right) \dot{e}_{\Delta \lambda}\left(T_{a m}\right)\right]
$$

Next, the measurements of the temperature of black body is repeated for $\boldsymbol{L}$ temperature levels of this body. On the basis of Eqs $(5,6)$ and the aforementioned measurement results a matrix of calibration factors $C_{l, i, j}$ defined by the relation (7) is created:

$$
C_{l, i, j}=\frac{s_{c o r}}{s_{\text {incor }}}=\frac{\varepsilon_{b b} \dot{e}_{\Delta \lambda}\left(T_{b b l}\right)+\left(1-\varepsilon_{b b}\right) \dot{e}_{\Delta \lambda}\left(T_{a m l}\right)}{\varepsilon_{b b} \dot{e}_{\Delta \lambda}\left(\bar{T}_{c l, i, j}\right)+\left(1-\varepsilon_{b b}\right) \dot{e}_{\Delta \lambda}\left(T_{a m l}\right)}
$$

where temperature $\overline{\boldsymbol{T}}_{\boldsymbol{c}} l, i, j$ representing the camera measurement result is an average value calculated on the basis of measurements repeated $\boldsymbol{M}$ times for each selected $\boldsymbol{l}$-th temperature value of the black body and is determined as follows:

$$
\bar{T}_{c \quad l, i, j}=\frac{1}{M} \sum_{m=1}^{M} T_{c} \quad l, i, j, m
$$

Next, after the creation of the matrix of calibration coefficients $C_{l, i, j}$ values, least squares adjustment method was applied to determine the coefficients $\boldsymbol{a}_{i, j}, \boldsymbol{b}_{\boldsymbol{i}, \boldsymbol{j}}$ of linear equations describing a dependence of calibration factors on the temperature $\boldsymbol{T}_{\boldsymbol{c}}$ within the temperature range $\boldsymbol{T}_{\boldsymbol{c} l=1} \div \boldsymbol{T}_{\boldsymbol{c} l=L}$ for each $\boldsymbol{i}, \boldsymbol{j}$-th pixel of the FPA detector:

$$
C_{i, j}=C\left(T_{c i, j}\right)=a_{i, j} T_{c i, j}+b_{i, j}
$$




\subsection{1/qirt.2016.066}

\section{Experiment description}

In the experiment the black body BB- 6 with plane testing surface and infrared camera ThermaCAM SC 2000 were used, Figs 2,3. The measurements have been carried out for several values of black body temperature taken from the range $15 \div 100^{\circ} \mathrm{C}$. The mentioned range was determined by the technical features of the black body device which we used.

Next, the obtained measurement results from the camera were compared with the preciously measured temperature of black body. On the basis of these data the temperature differences for each pixel of the infrared camera detector were calculated. The gathered temperature differences were used for the determination of mathematical relationships individually for each pixel of the detector array. Then, to improve the quality of measurement results, these relationships can be used for the correction of results produced directly by the camera. Additionally, various statistical quantities were calculated which were useful for the evaluation of camera detector measurement features. To realize this process the MATLAB package was employed.

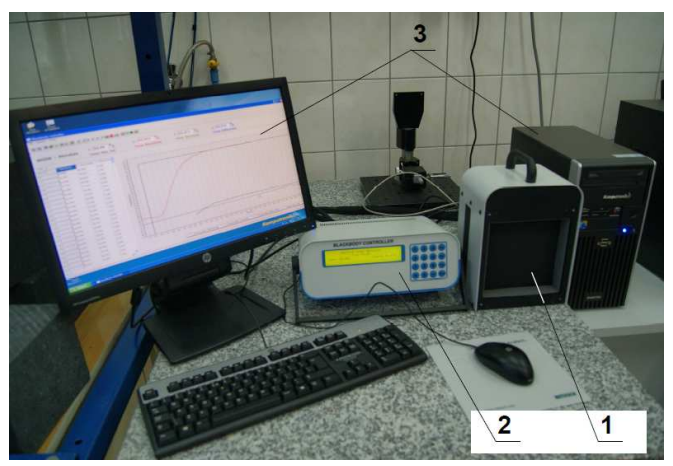

Fig. 2. The black body model BB-6, 1-black body surface (emitter of thermal radiation), 2-black body controller, 3-controlling computer

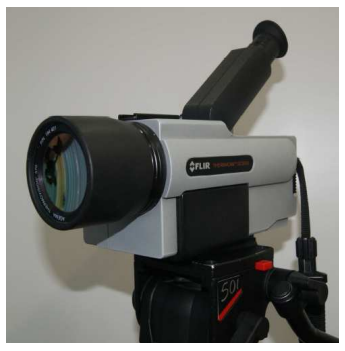

Fig. 3. The examined infrared camera

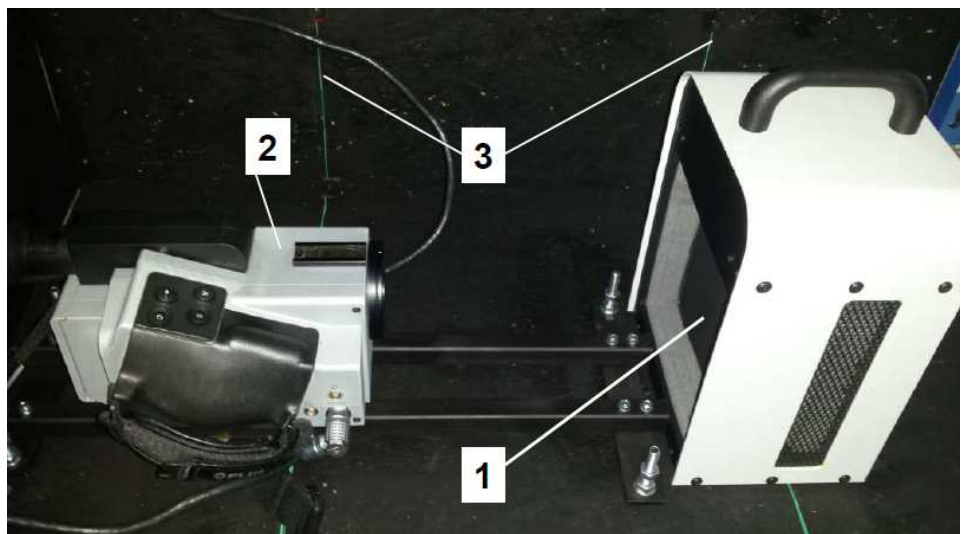

Fig. 4. The infrared camera and the black body in an isothermal chamber, 1-black body, 2-infrared camera, 3-thermocouples for measurement of the chamber walls temperature 


\subsection{1/qirt.2016.066}

\section{Correction of measurement results}

After determination of the matrix of coefficients $C_{l, i, j}$ on the basis of Eq. (9), for each pixel the corrected values $\boldsymbol{T}_{\boldsymbol{o b r} \boldsymbol{i}, \boldsymbol{j}}$ of the considered object temperature can be calculated. These values can be obtained on the basis of Eq. (10):

$$
\dot{e}_{\Delta \lambda}\left(T_{o b r i, j}\right)=C_{i, j}\left(T_{o b c i, j}\right)\left\{\dot{e}_{\Delta \lambda}\left(T_{o b c i, j}\right)+\frac{1-\varepsilon_{o b}}{\varepsilon_{o b}} \dot{e}_{\Delta \lambda}\left(T_{a m}\right)\right\}-\frac{1-\varepsilon_{o b}}{\varepsilon_{o b}} \dot{e}_{\Delta \lambda}\left(T_{a m}\right)
$$

where $\boldsymbol{T}_{\boldsymbol{o b} \boldsymbol{c} i, j}$ is the considered object temperature value shown by the camera for $\boldsymbol{i}, \boldsymbol{j}$-th pixel of the camera detector. The desired values of the temperature $\boldsymbol{T}_{\boldsymbol{o b r} \boldsymbol{i}, j}$ occur in the implicit form in expression $\dot{\boldsymbol{e}}_{\Delta \lambda}\left(\boldsymbol{T}_{\boldsymbol{o b r} \boldsymbol{i}, j}\right)$. The total value of this expression is known and therefore to calculate the desired temperature from relation $(10)$ the Eqs $(1,2)$ should be additionally used.

\section{Calculated parameters and calculation results}

To evaluate the effectiveness of external calibration procedure and the quality of measurement results produced by the IR camera for all pixels of FPA detector the temperature differences defined by the relation (11) were calculated:

$$
\delta T_{l, i, j}=\bar{T}_{c} l, i, j-T_{b b l}
$$

The calculation results have been shown for selected black body temperature levels, in Fig. 5 . These results are useful for the evaluation of measurement results quality obtained by means of the considered camera.
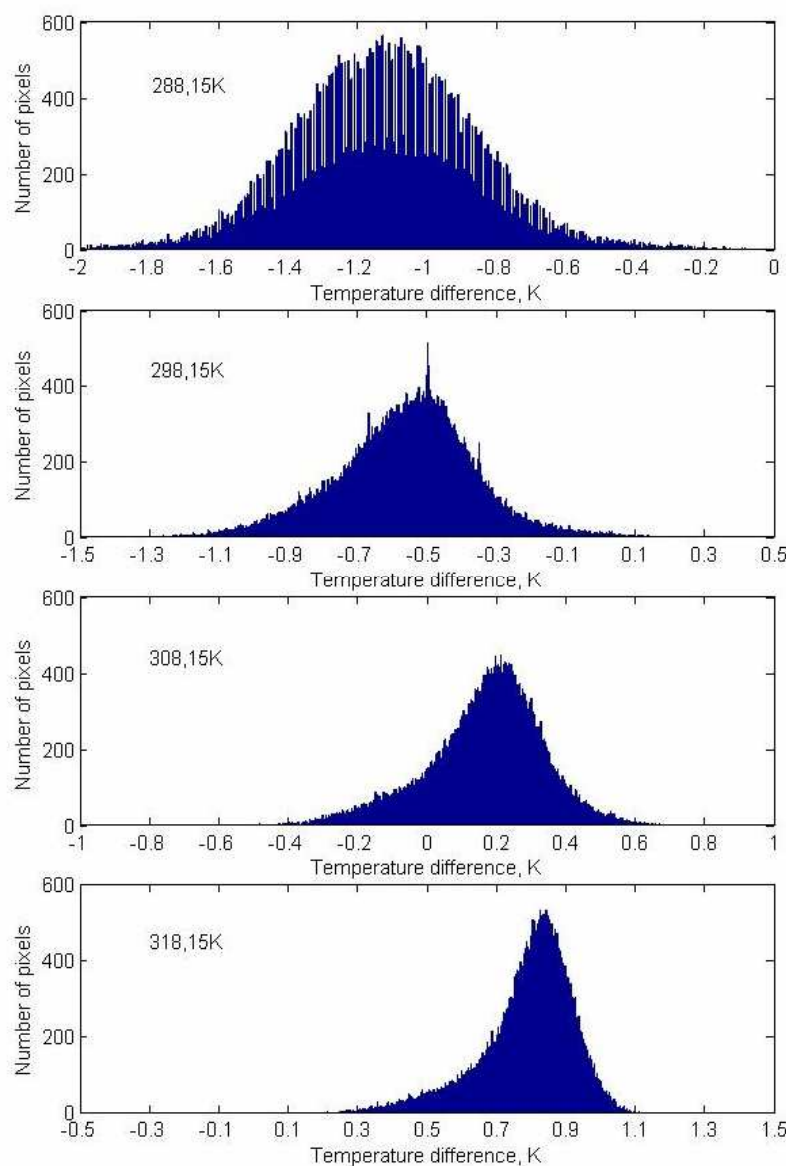
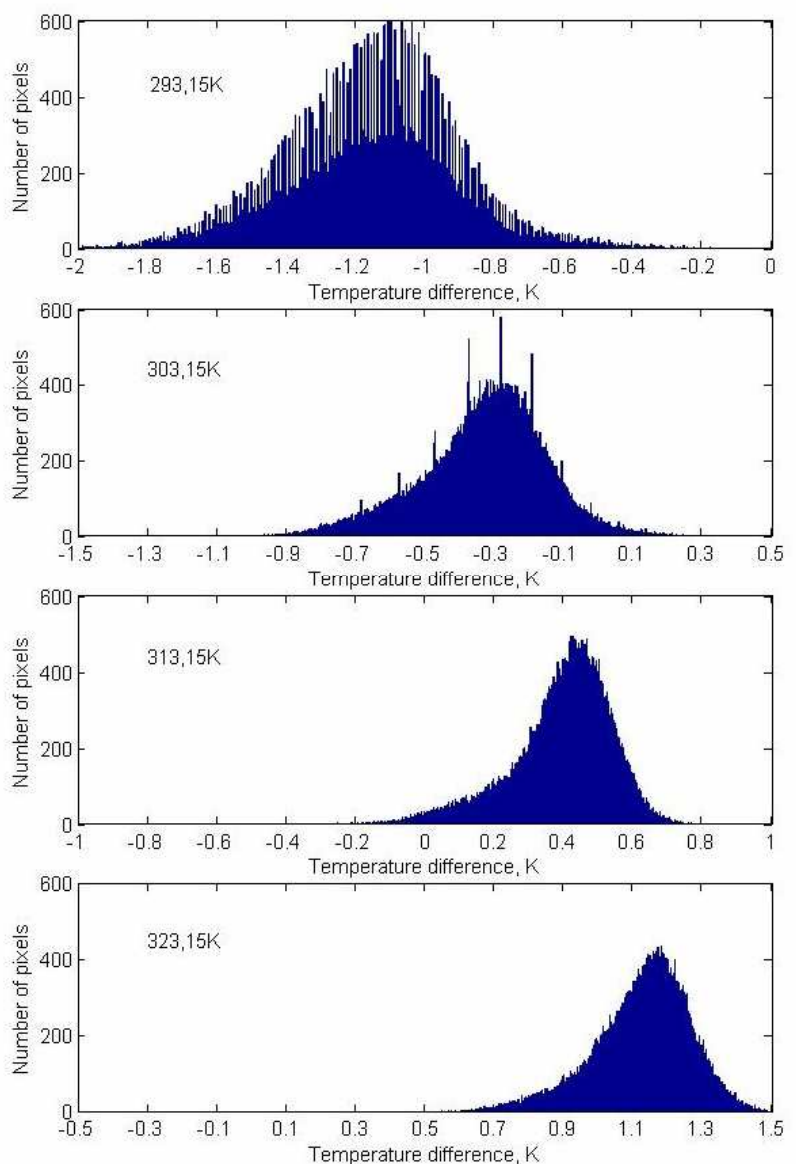

Fig. 5. Histograms of differences between the temperature measured by the IR camera and indicated by the BB before correction, (Eq. 11) 


\subsection{1/qirt.2016.066}

The similar temperature discrepancies can be calculated for the corrected values $\boldsymbol{T}_{\boldsymbol{b} \boldsymbol{r} \boldsymbol{l}, \boldsymbol{i}, \boldsymbol{j}}$ of the BB temperature measured by the IR camera:

$$
\delta T_{r l, i, j}=T_{b r l, i, j}-T_{b b l}
$$

In this case the obtained results testify to the quality of calibration method used for the improvement of the quality of measurement results obtained by means of the examined IR camera.

The temperature values $\boldsymbol{T}_{\boldsymbol{b} \boldsymbol{r} l, i, j}$ are calculated from the relation (13) resulting from Eq. (10):

$$
\dot{e}_{\Delta \lambda}\left(T_{b r l, i, j}\right)=C_{i, j}\left(\bar{T}_{c l, i, j}\right)\left\{\dot{e}_{\Delta \lambda}\left(\bar{T}_{c l, i, j}\right)+\frac{1-\varepsilon_{b b}}{\varepsilon_{b b}} \dot{e}_{\Delta \lambda}\left(T_{a m l}\right)\right\}-\frac{1-\varepsilon_{b b}}{\varepsilon_{b b}} \dot{e}_{\Delta \lambda}\left(T_{a m l}\right)
$$

where the values of coefficient $C_{i, j}\left(\bar{T}_{c} l, i, j\right)$ are calculated on the basis of known relation (9). The calculation results have been presented in Fig. 6.
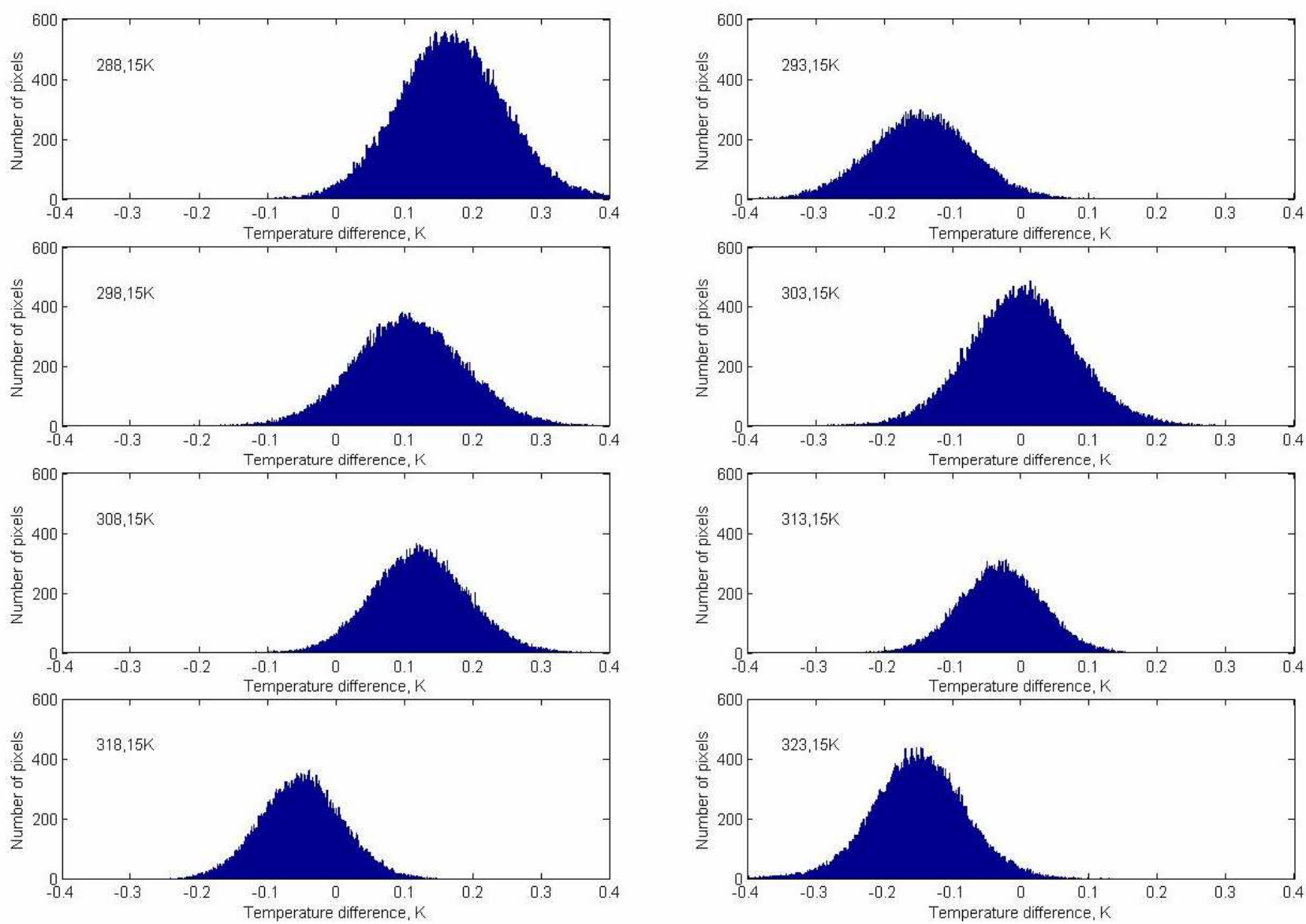

Fig. 6. Histograms of differences between the temperature measured by the IR camera and indicated by the BB after correction, (Eq. 12)

In Fig. 7 a histogram presenting dispersion of correction coefficient $\boldsymbol{C}$ defined by expression (7) has been shown. This diagram presents the differences in terms of conversion quality of thermal radiation into a measurement signal by the infrared detector for all pixels of the detector matrix. 

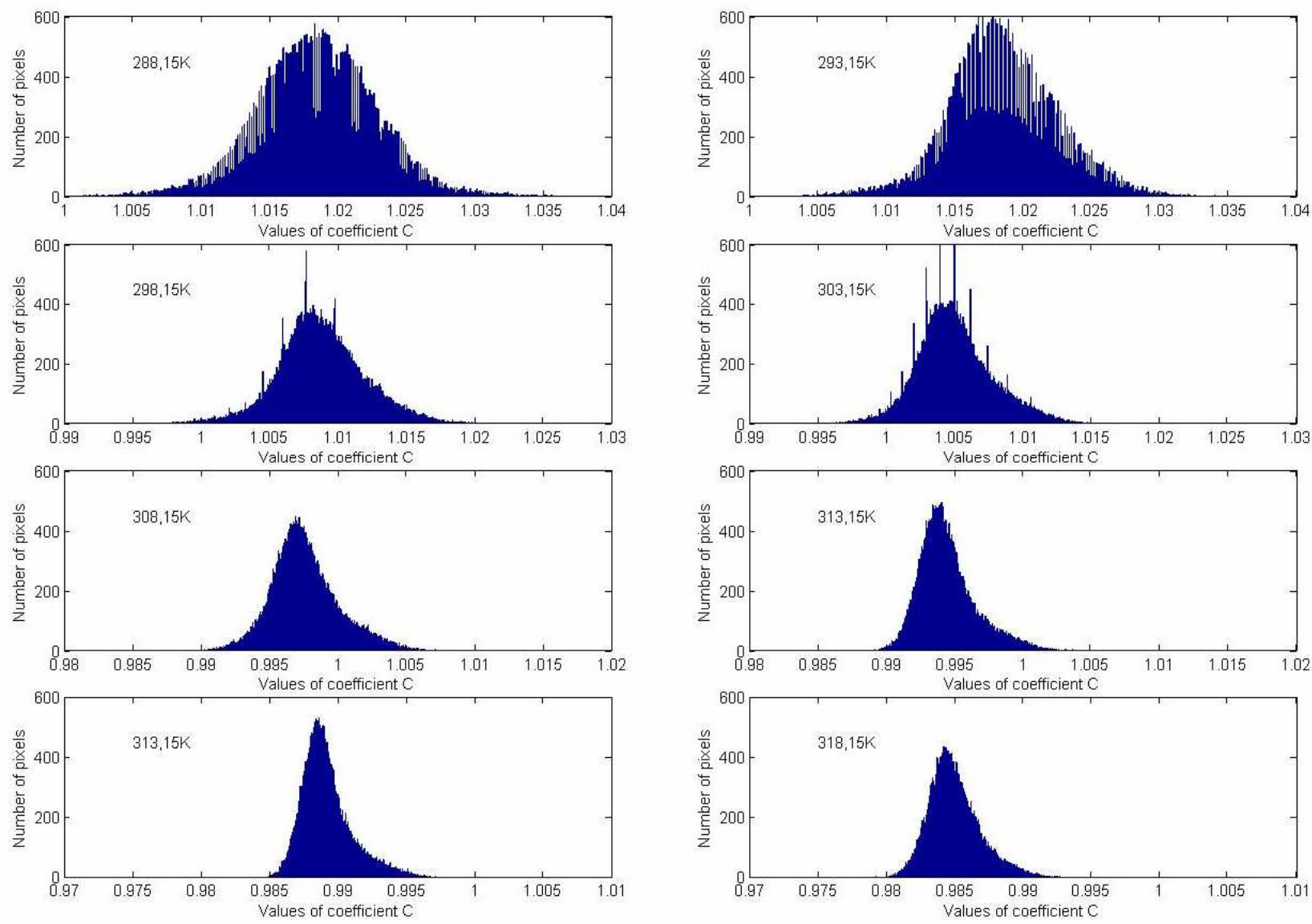

Fig. 7. Histogram presenting the dispersion of value of the correction coefficient $\boldsymbol{C}$

Additionally, in Fig. 8 are presented two samples of correction line determined for two pixels of FPA detector.
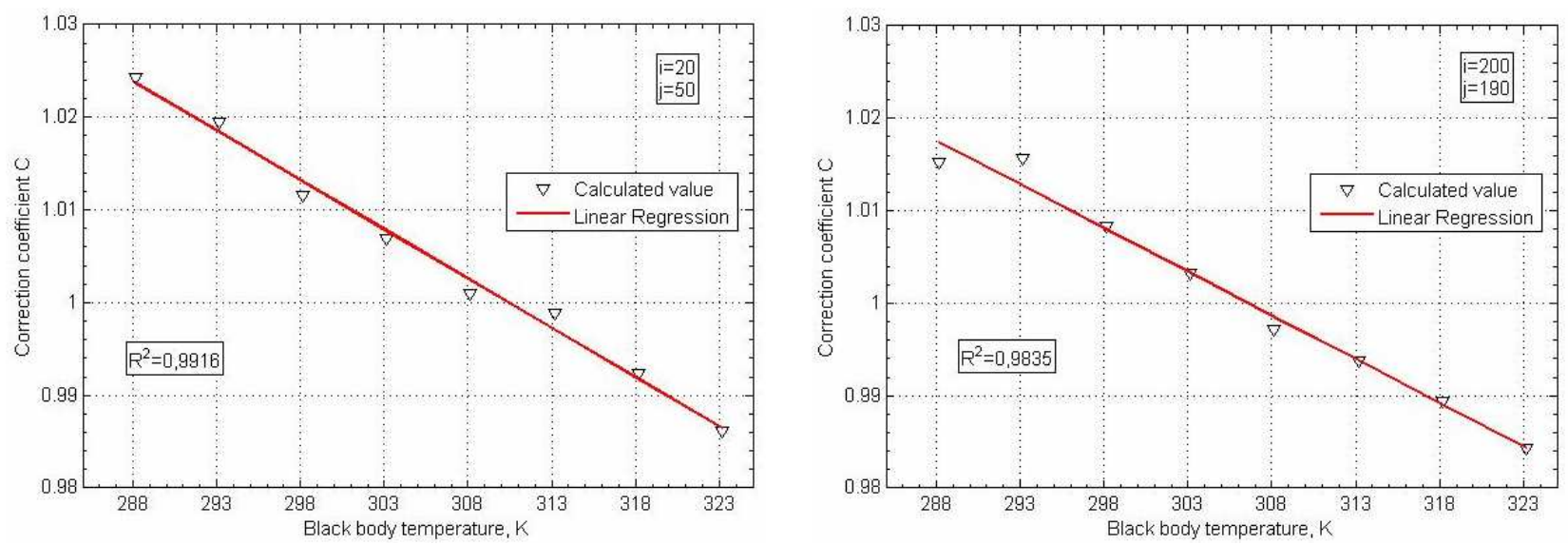

Fig. 8. Correction lines of coefficient $\boldsymbol{C}$ for specified in legend coordinates of pixels of FPA detector

As it is described, for all pixels of IR camera detector the correction lines expressing the changes of correction coefficient according to the measured temperature by means of the least squares adjustment method have been determined. Simultaneously, the values of a correlation coefficient have been calculated. The value of this coefficient expresses the quality of the fitting of the determined line to the considered set of points. The obtained results are shown in Fig. 9. The fitting of correction lines is quite satisfactory, because for most pixels of the camera detector the correlation coefficients take values greater than 0.95, on the average 0.97, Fig. 9. 


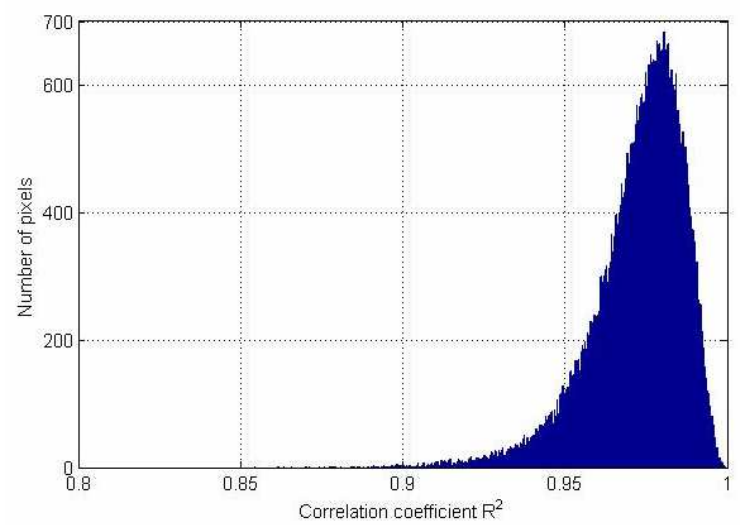

Fig. 9. Histogram of values of correlation coefficient $\boldsymbol{R}^{2}$ for the determination of correction coefficient $\boldsymbol{C}$

\section{Verification of method}

To verify the developed method, an additional measurement series using the examined IR camera and the black body model was made. The experiment was carried out for the temperature of black body amounting to $36.6{ }^{\circ} \mathrm{C}$ (i.e. $309.75 \mathrm{~K}$ ). Next, the obtained measurement results have been corrected with the use of Eq. (13) and for all pixels of IR detector the temperature differences between measurements and the black body temperature before and after correction have been calculated according to Eqs $(14,15)$ :

$$
\begin{aligned}
\delta T_{b c, i, j} & =T_{b c i, j}-T_{b b} \\
\delta T_{b r, i, j} & =T_{b r i, j}-T_{b b}
\end{aligned}
$$

Next, the obtained results have been presented in a form of histograms (in Fig. 10), and as visual matrix images, Fig. 11.

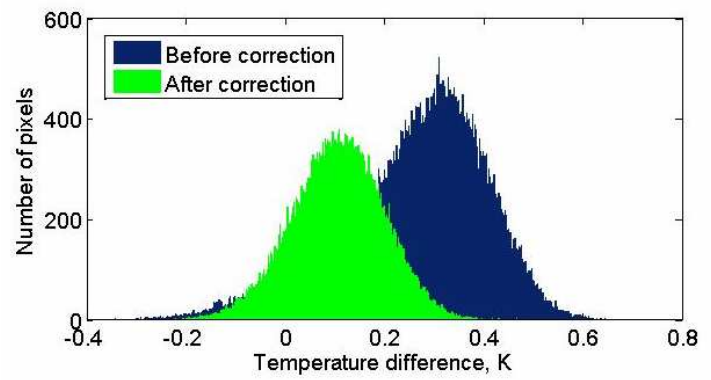

Fig. 10. Histogram of the temperature differences between the temperature indicated by the camera and the black body before and after applying the correction procedure 

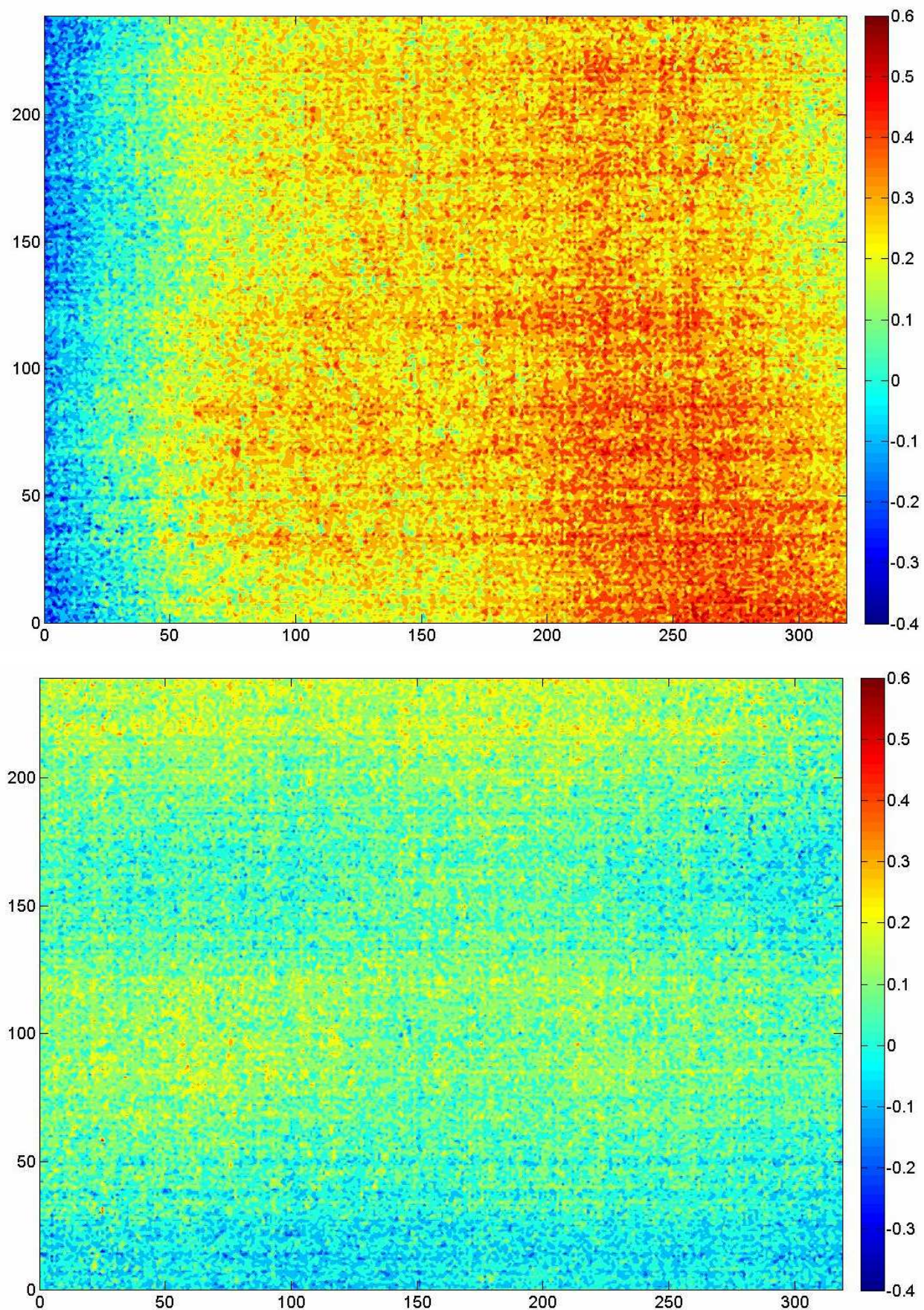

Fig. 11. Visual image of distribution of temperature differences between the temperature indicated by the camera and the black body measuring system before (top) and after (bottom) applying the correction procedure for the examined infrared camera

\section{Sample of application of the developed method}

To check the usefulness of the developed method the data recorded by IR camera for a human palm have been processed by means of the described method. The obtained results are presented in Fig. 12 where can be seen the visual images of a palm before and after applying the presented correction method. The differences between both images are rather small due to the applying of relatively wide temperature span $(6 \mathrm{~K})$ in the images, however they are noticeable. 

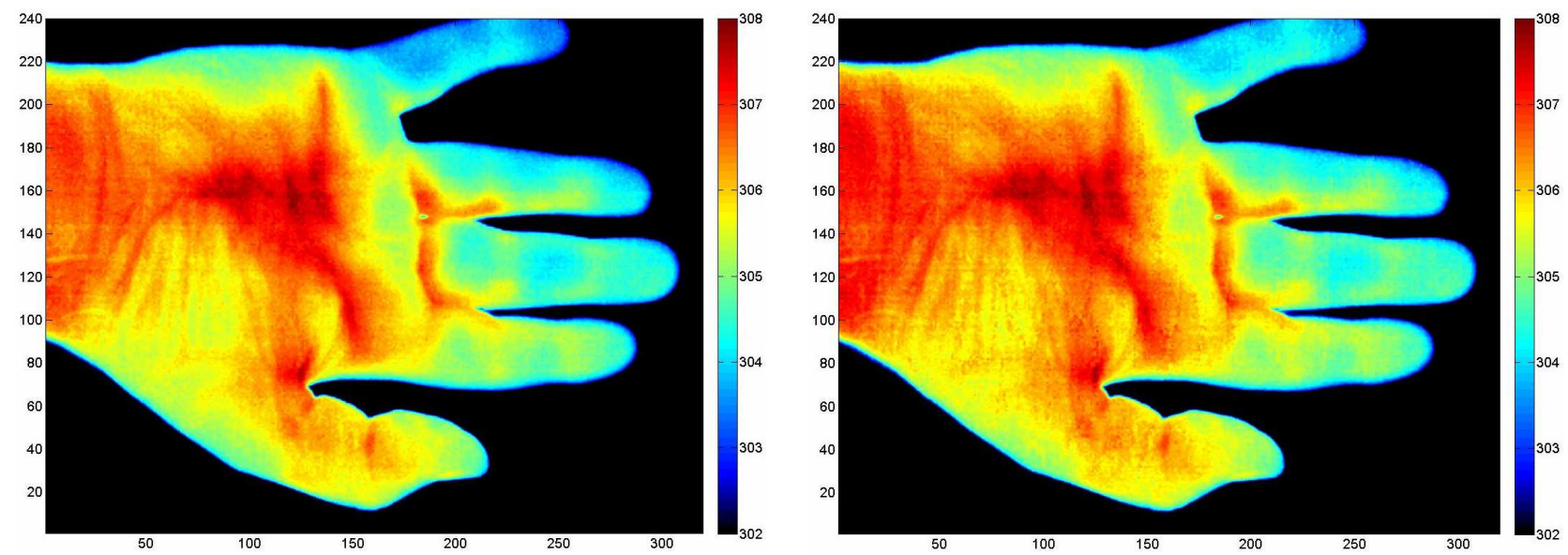

Fig. 12. Visual images of a palm generated by means of Matlab, before (on the left) and after applying (on the right) the presented correction method

\section{Final remarks and conclusions}

In the paper the method of improving the accuracy of temperature measurement results obtained by means of an infrared camera equipped with FPA detector is presented. The typical accuracy of infrared cameras amounts to $\pm 2 \mathrm{~K}$ or even $\pm 2 \%$ of the measured value of temperature. Very often a better accuracy of the measurements is required, e.g. during the measurements of human body temperature. To improve the measurement accuracy an external correction of obtained results on the basis of comparison measurement of black body temperature and next with use of MATLAB package has been described.

The developed method allows to improve locally in term of temperature range, the accuracy of measurement results recorded by the IR camera. It is expected that in the case of using the black body model of high accuracy in this calibration procedure, the reaching of measurement accuracy close to detector thermal sensitivity is possible.

In the work different diagrams which are useful for the evaluation of IR camera measurement accuracy and its operation have been worked out and presented. It is expected that the proposed method will be very effective for narrow measurement ranges of temperature.

\section{Acknowledgements}

The research has been supported by National Science Centre within OPUS scheme, under contract UMO-2013/11/B/ST8/00268 and SONATA scheme under contract UMO-2014/15/D/ST8/02620.

\section{REFERENCES}

[1] Howell J.R., Siegel R., Mengüç M.P., Thermal radiation heat transfer. CRC Press Taylor\&Francis Group, New York 2011.

[2] Modest M.,F., Radiative heat transfer. McGraw-Hill, Inc., New York 1993.

[3] Technical Documentation ThermaCAM SC2000, FLIR.

[4] Minkina W., Dudzik S., Infrared Thermography - Errors and Uncertainties. Wiley \& Sons, UK; 2009.

[5] Olbrycht R., Więcek B., Correction of microbolometer detector temperature drift, Measurement Automation and Monitoring, vol. 55 (2009), No 11, pp. 890-893.

[6] Rainieri S., Pagliarini G., Data processing technique applied to the calibration of a high performance FPA infrared camera, Infrared Physics \& Technology, Vol. 43 (2002), pp.345-351.

[7] Kruczek T., "Analysis of the influence of external conditions on thermovision measurement results", Procedings of 5-th Conference Thermometry and Thermography in Infrared, Ustroń (Poland), 2002, pp.327-332, (in Polish).

[8] Kruczek T., Conditions for the use of infrared camera diagnostics in energy auditing of the objects exposed to open air space at isothermal sky. Archives of Thermodynamics, vol. 36, No. 1, pp.67-82, 2015.

[9] Kruczek T., Use of infrared camera in energy diagnostics of the objects placed in open air space in particular at non-isothermal sky. Energy, vol. 91, pp.35-47, 2015. 